\title{
Représentation(s), manifestation(s) et illustration(s) de la femme
}

La publication du Deuxième sexe en 1949, marque un moment clé des études féministes même si ce texte majeur de Simone de Beauvoir est parfois contesté. La même période voit aussi l'arrivée de la deuxième vague du féminisme contemporain, où les féministes appuient leurs actions concrètes sur une réflexion théorique particulièrement vive et élaborée sur la sexualité, les droits de la femme dans la famille et le lieu de travail, et son droit à la reproduction: The Feminine Mystique (1963) de Betty Friedan, The Female Eunuch (1970) de Germaine Greer, The Dialectics of Sex (1971) de Shulamith Firestone, et le Sexual Politics (1972) de Kate Millet. Ces développements théoriques leur ont permis de mieux définir tant le passé et les acquis des mouvements féminins que certaines contradictions entre l'idéologie et la pratique des mouvements (Kaplan).

Si Simone de Beauvoir et ses émules considèrent la socialisation de la féminité comme une oppression dont la femme est victime, d'autres, comme Camille Paglia, valoriseront ce changement social et verront en elle une arme idéale pour la femme. Pour Paglia, la socialisation de la sexualité féminine, avec les notions de modestie et de vertu qu'on impose aux femmes, leur accorde aussi la liberté de refus ou de soumission.Si le mythe de l'éternel féminin, mis à mal par Simone de Beauvoir, paraît plus que jamais désuet dans notre société moderne, il n'en reste pas moins que les femmes, notamment par leur corps, sont toujours perçues comme un "fétiche de la représentation" (Butler 25) sur lequel se greffe souvent un héritage biblique à la vie dure où la femme, créée à partir de la côte d'Adam, reste un être secondaire.

Par ses représentations, manifestations ou encore illustrations, il faut donc comprendre la femme comme un miroir autant qu'une projection de sa place et de son rôle dans la société. La femme mise en images reste un élément privilégié autant en littérature qu'en arts visuels. En quoi donc les représentations de la femme illustrent-elles la condition féminine et son évolution dans les lettres et les arts visuels ? De quelle manière et à quelle(s) fin(s) est représentée la femme ? Peut-on s'emparer de la femme comme objet représentatif? Les arts contemporains ont-ils fait basculer le statut de la femme d'objet désiré à celui de sujet désirant ? Nous retrouvons ces questionnements dans les trois champs d'étude de ce numéro, à savoir la littérature africaine francophone, les arts du spectacle et les arts visuels.

Dans la «Représentation du corps féminin dans le roman africain francophone : Les cas de Salimata dans Les Soleils des Indépendances d'Ahmadou Kourouma et Perpétue dans Perpétue et l'habitude du malheur de Mongo Beti », Ibrahim Boumazzou propose une étude sur la condition de la femme en Afrique (particulièrement en Côte-D'ivoire et au Cameroun), aux lendemains des indépendances. S'appuyant sur des analyses minutieuses des parcours de vie des protagonistes (l'excision, le viol, la mise en vente à travers la coutume de la dot, et la soumission conjugale), de leurs sensations individuelles (douleur, horreur, déchirement, désespoir, etc.) et de la signification symbolique des mots-clés (impureté, rouge, objet, liberté, etc.), Boumazzou démontre que le corps féminin est prisonnier et victime d'un ensemble de stéréotypes patriarcaux (traditions, coutumes, religions) qui définissent les femmes par leur sexualité et entravent l'épanouissement et l'autonomie de celles-ci. 
Tournons nos regards vers le continent de l'Amérique du Nord et le domaine des arts du spectacle. Une recherche documentée en prenant compte des visions diversifiées (thématique, technique, historique et sociale), l'article « Entre présentation et représentation : le corps à travers l'œil de la caméra de Carolee Schneemann » de Marie-Laure Delaporte s'intéresse à la construction d'un discours aristico-féministe dans Meat Joy (1964) et Fuses (1964-1967). L'auteure s'attache à mettre au jour la fonction du corps qui se fait matériau et instrument de vision, créateur et objet de la création, permettant aux spectateurs de confronter leur identité, de prendre en conscience de l'altérité et de faciliter l'acceptation d'autrui. Elle souligne également la valeur de la nudité artistique chez Schneemann, pour qui il s'agit de la revendication de la liberté ainsi que de la dénonciation de la violence dirigée vers les femmes. Tout en explorant l'influence qu'exercent les réalisateurs tels que Maya Dren et Stan Brakhage sur l'artiste, Delaporte identifie le rôle que joue la caméra par sa capacité de participer au mouvement engendré par le corps, et de fournir une documentation de cette énergie visuelle aux qualités expressives et expressionnistes.

Il est également question du corps de la femme dans « Le "monstrueux corporel" : Phèdre de Jean Racine mis en scène par Patrice Chéreau » de Marine Deregnoncourt. C'est un théâtre de corps et une scénographie révélant les profondeurs "sauvages" du texte racinien que Chéreau propose au spectateur. Chez Chéreau, Phèdre explore et expose un monstrueux multidimensionnel, à la fois physique, moral et verbal, qui s'esquisse sous les non-dits et dans la violence du désir et de l'interdit sous-tendant les actes et les paroles des personnages.

Autre art propice à la mise en images du corps de la femme, le cinéma a bien souvent été accusé d'hétérocentrisme, ce que Riad Sattouff semble se faire une joie de déconstruire dans son film Jacky au royaume des filles selon Nathalie Rouphael dans « La représentation inversée des valeurs et normes féminines dans Jacky au royaume des filles de Riad Sattouf ». Proposant une approche résolument transdiciplinaire ancrée dans les études culturelles et au carrefour des Queer studies, des études de Simone de Beauvoir, Judith Butler et Kimberlé Crenshaw, Rouphael s'intéresse à la déconstruction des normes hétérocentristes dans le film de Sattouff sous le prisme de la "théorie du reflet" ou du "miroir inversé"; le cinéma, miroir de la réalité, reflète tout autant qu'il renverse les normes en cours dans notre société.

Pour finir, cette quatrième livraison de Convergences francophones inaugure une section Varia avec un article d'Olga Kulagina sur les représentations de l'ailleurs et de l'autre dans deux récits de voyages du XVIIe siècle. L'auteure compare les procédés d'écriture à l'œuvre chez Philippe Avril et Jean-Baptiste Tavernier pour montrer que si la divergence de visée des voyageurs peut bien conduire à la priorisation de certaines thématiques, elle se retrouve parfois aplanie par le filtre de leur culture d'origine dans l'inventaire ethnographique développé dans le récit.

Pour leur soutien, nous tenons aussi à remercier Azouz Ali Ahmed, Mark Andrews, Stéphanie Bérard, Justin Bisanswa, Véronique Bonnet, Isabelle Cata, Romain Chareyron, Yves Chemla, Claire Dehon, Frédérique Devaux, Danielle Dumont, Carole Edwards, Pascal Fobah Eblin, Karen Ferreira-Meyers, 
Gwénaëlle Le Gras, Michèle Longino, Hélène Marquié, Maribel Peñalver, Vincent Simedoh, Alexandre Stroev, Guy Baudelaire Tegomo et Rainer Zaiser

Nous tenons également à remercier Xiaoyin Liu, créatrice de l'illustration utilisée pour la couverture de ce numéro. Enfant, Xiaoyin Liu voulait devenir artiste. Découragée par son entourage qui lui prédisait un avenir sombre, elle a poursuivi des études en design industriel. Elle termine actuellement sa maîtrise en design environnemental à l'université de Calgary et elle s'intéresse particulièrement au design de produits durables. Liu rassemble et promeut constamment le design, les pratiques et le mode de vie durables à travers des approches artistiques. Ses œuvres se trouvent sur compte Instagram sustainablestory et sur son compte public Wechat sustainablestory.
Sur un fond rouge symbolisant le sang, la violence et la tragédie des violences faites aux femmes, se détache le visage d'une femme aux yeux couverts et sans bouche, témoignant de son confinement à la sphère privée et d'une parole muselée. Image du passé ou bien encore d'actualité ? De plus près, deux silhouettes se détachent dans une scène de lutte : celle d'un homme en bleu et noir et celle d'une femme en rose et gris. Cette scène correspond bien au quotidien de nos sociétés patriarcales. Pour Liu, l'imbrication des deux scènes illustre deux états de la femme indissociables dans la représentation de la femme aujourd'hui.

\section{Bibliographie}

Beauvoir de, Simone. Le deuxième sexe. Paris: Gallimard, 1949.

Butler, Judith. Gender Trouble. Feminism and Subversion of Identity. London/New York: Routledge, 1999.

Kaplan, Gisela. Contemporary Western European Feminism. London: Allen \& Unwin, 1992. 